International Journal on AdHoc Networking Systems (IJANS) Vol. 8, No. 1, January 2018

\title{
ADAPTIVE ARRAY BEAMFORMING USING AN ENHANCED RLS ALGORITHM
}

\author{
Peter Chuku, Thomas Olwal and Karim Djouani \\ Department of Electrical Engineering, FSATI \\ Tshwane University of Technology \\ Staatsatilarie Road, Pretoria West, South Africa
}

\begin{abstract}
In recent times, the use of Smart Antennas (SAs) in wireless communication systems has greatly increased. This is because of their ability to increase the coverage and capacity of a communication network. There are two main functions which SAs can perform. The first one is to ensure that the signal has a proper direction of arrival (DOA) and the second function is to achieve the desired beamforming goals. That is, the SAs can produce the main beams towards a desired user and null the beam in the direction of interfering signals at the same time. They are able to achieve this by the use of beamforming algorithms. The performance of an enhanced Recursive Least Squares (RLS) algorithm which takes into account an enhanced gain factor has been evaluated in this paper for adaptive array beamforming. Simulation results have shown that the enhanced RLS algorithm reduces a mean square error (MSE), smoothens a filter output and improves the Signal to Noise Ratio (SNR). The impact of these results can imply an increased range of beamforming and directivity of the smart antenna over a long range communication network.
\end{abstract}

\section{KEYWORDS}

Smart antennas; direction of arrival; adaptive arrays.

\section{INTRODUCTION}

An antenna is an electronic device used to transmit and or receive electromagnetic waves [1]. For broadcasting purposes, an array of antennas is used to produce optimum gain and directivity at a particular direction whilst at the same time, produces minimum gain and directivity in other directions. The radiation patterns of an antenna illustrate areas of gain (main lobes) or attenuation (nulls) for the signals it transmits or receives [2].

Recently, smart antennas (SA) also known as adaptive antennas have become a fundamental aspect for most wireless and other long range communications applications [3]. SAs are utilised for the purpose of spatial filtering in order to receive desired signals coming from a specific source while reducing the reception of unwanted signals coming from other directions.

In some antenna applications, it is important that the receiver is aware of the direction of arrival of the transmitter's signal; that way, the receiver will have the capability to steer the signal's beam in order to maximise the signal in that specific direction. The ability for an antenna to adjust (steer) the radiation pattern towards the transmitter is called Beamforming [4]. Beamforming is

DOI: $10.5121 /$ ijans.2018.8101 
International Journal on AdHoc Networking Systems (IJANS) Vol. 8, No. 1, January 2018

central to all antenna arrays. Beamforming can be applied by either changing the amplitude of excitation or altering the phase of excitation which is responsible for steering the beam.

In order to achieve optimum gain and directivity in phased array antennas, the elements are connected to a digital signal processor which steers the beam automatically [2]. The technique required to achieve maximum directivity in the desired direction of a beam by making use of digital signal processing is called digital beamforming [5].In SAs, it takes a process to determine the complex weights and control the output in order to get the specified direction of the beam. This process is known as adaptive digital beamforming. In adaptive beamforming, many adaptive filtering techniques are used in determining the complex weights of SAs [6]. Figure 1 below illustrates adaptive beamforming; the prominent beam formed is utilised by the user whereas, the interfering signals are nulled.

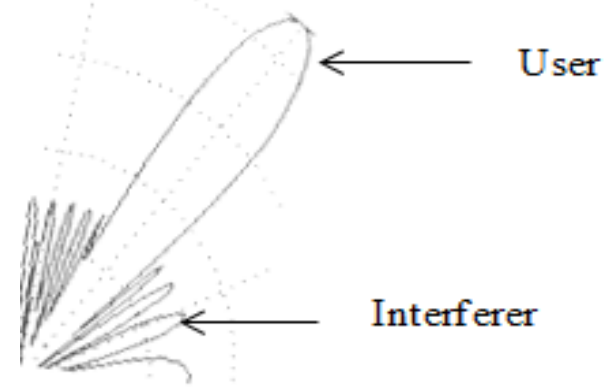

Figure 1: Adaptive beamforming [7]

One of the adaptive filtering techniques that can be used to determine complex weights is the recursive least squares (RLS). Other adaptive filters are the least mean squares (LMS), constant modulus algorithm (CMA), etc. [8 and 9]. The performance of most adaptive filters lies on the condition of their signal and filter order. However, the performance of the RLS algorithm is linked to its forgetting factor $\lambda$. As has been stated by authors in [8], a complex weight is the combination of the relative amplitude and phase shift for each antenna element. When compared to other adaptive filters or algorithms, the RLS algorithm is more superior in its filtering abilities because of its convergence speed and smaller mean square error (MSE) [9].

Several filtering techniques have been proposed by past authors [10] to enhance the performance of the RLS algorithm but these improvements came with the cost of computational complexity. Thus, our paper presents an enhanced RLS based algorithm that improves the gain and directivity of beamforming over long range communication networks. This study is founded upon the fact that the gain vector $\mathrm{k}(\mathrm{n})$ is a vital entity in the performance of the RLS algorithm which will have a resultant effect in the adaptive array beamforming. We infer that an enhancement of the gain vector $\mathrm{k}(\mathrm{n})$ will lead to a significant improvement in the convergence rate of the RLS algorithm.

The rest of this paper has been structured as follows: section II presents literature review on related work on beamforming and the RLS algorithm, in section III, we focus on the system model of our enhanced RLS algorithm, section IV shows the performance evaluation and results of our enhanced RLS algorithm and section V is a summary of our work. Sections VI and VII, indicates the acknowledgement and references respectively. 
International Journal on AdHoc Networking Systems (IJANS) Vol. 8, No. 1, January 2018

\section{RELATED WORK}

By our definition, beamforming is a method of transmitting or receiving signals by directing the signals to a preferred or particular direction. Authors in [7] defined beamforming as a technique of using a fixed or adaptive beam pattern to electronically control the directionality of an array of transducers (either transmitting or receiving). In actual sense, beamforming takes into account signals coming into SA arrays from a desired location whilst nullifying or reducing the effect of interfering signals coming from other directions [8]. One of the functions of beamforming is spatial filtering; that is beamforming can increase the sensitivity of the receiver towards the direction of its desired signal and at the same time, reducing the receiver's sensitivity towards the direction of noise [9]. Beamforming can be classified into analog and digital beamforming [7].

There are two types of beamforming: fixed (conventional) and adaptive (switched) beamforming. The fixed beamforming makes use of constant weights and time delays to perform its processing. Whereas in the adaptive beamforming, adaptive noise cancellation is performed automatically of which optimal signals are derived from the direction of a steering antenna. The adaptive beamforming technique does not require a maximum signal in order to get an optimum beamforming output but rather, it nulls the effects of noisy signals from undesired directions [10]. Hence, an adaptive beamformer is a beamforming radar system that performs signal processing on a SA system which does not require steering [11].

An antenna array is a configuration of multiple antenna elements which are arranged in such a way that they give a particular radiation pattern [12]. It employs the use of adaptive beamforming algorithms in order to recognize, track and suppress interfering signals. The radiation pattern of a SA is optimized when a set of weights are adjusted by combining the signals incident on the antenna when the direction of arrival (DOA) is known [13]. A combination of the relative amplitude and phase shift for each SA element is called a complex weight [14]. The use of adaptive algorithm is expedient for the calculation of continuously updated weights. There are several types of adaptive algorithms of which the RLS is one of them. RLS uses weight updating techniques. The filter order and signal condition of adaptive algorithms has an effect on their performance. Therefore in beamforming, the radiation pattern of a SA is manipulated through various adaptive algorithms. The figure below (fig. 2) illustrates how adaptive algorithms are used to achieve adaptive filtering.

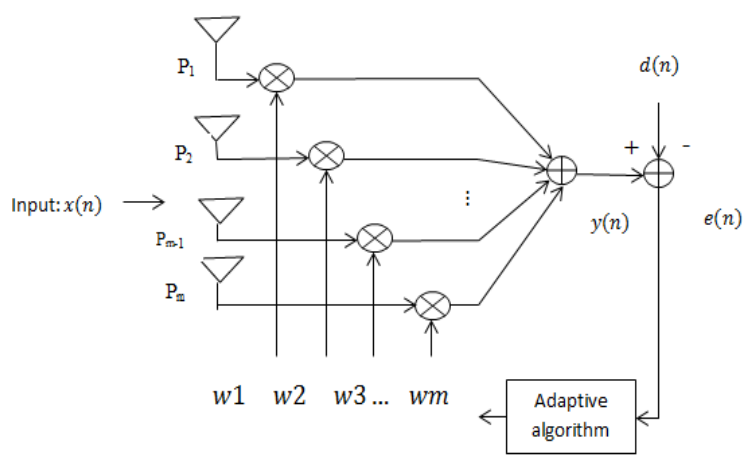

Figure 1: Adaptive filtering 
International Journal on AdHoc Networking Systems (IJANS) Vol. 8, No. 1, January 2018

The performance of RLS algorithm depends on the value of $\lambda$ which is known as the forgetting factor or sometimes called the exponential weighting factor [15]. The RLS algorithm recursively finds coefficients of the filter that minimize a weighted linear least squares function which are related to the input signals of the filter. This in turn, reduces the mean square error (MSE). In deriving the RLS algorithm, the input signals are considered deterministic (the performance index is the sum of weighted error squares for a given data) whereas, for the least mean squares (LMS) algorithm, the input signals are stochastic. [16]. Compared to other adaptive algorithms, the RLS algorithm exhibits the fastest convergence. This is because the RLS algorithm whitens the input data by using the data's inverse correlation matrix which is assumed to be of zero mean [17].

In summary, the purpose of adaptive beamforming is to form narrow beams towards desired users while at the same time, creating nulls at interfering signals by means of adjusting the weights vector of the beamformer. Updating of weights vector increases the performance of a communication channel by enhancing the directivity of a SA array.

\section{SYSTEM MODEL}

RLS algorithm is known for its recursive filtering techniques by adapting the weights $w(n)$ of the filter recursively. And in order to adapt the weights, the error signal $e(n)$ and the gain factor $k(n)$ must be determined. The error signal is determined by calculating the difference between the desired signal $d(n)$ and the output signal $y(n)$ of the filter. The number of antenna elements must we known. The beam pattern is then sampled. Prior to sampling of the beam pattern, the following variables must be entered:

Sample size and the phase shift of the weight $\theta_{k}$.

Afterwards, the beam pattern is then calculated by:

- Deciding on the number of antenna elements N

- Determining the angle of the desired signal $\theta_{n}$

- Setting the relative amplitude of the weight $a_{0}$ which is denoted by:

$$
a_{0}=e^{(-j * \pi *(0: N-1) * \sin (\pi 0))}
$$

Where $0: \mathrm{N}-1$ is the antenna array factor and

$\mathrm{J}$ is a constant $(\sqrt{ }(-1))$

The of this section is to determine an improved value of the gain factor $k(n)$ which will yield better the weights $w_{n}$ of the RLS filter.

Formation of beam patterns involve complex weights multiplication.

$$
w_{k}=a_{k} \cos \left(\theta_{k}\right)+j a_{k} \sin \left(\theta_{k}\right)
$$

Where $w_{k}$ is the complex weight of the $k^{t h}$ antenna element. 
International Journal on AdHoc Networking Systems (IJANS) Vol. 8, No. 1, January 2018

After the formation of the beam patterns, the variables that constitute the weight vector for the RLS algorithm are then entered to the filter:

$$
w(n)=w(n-1)+k(\mathrm{n}) e(n),
$$

where the error signale $(n)$ is calculated as

$$
e(n)=d(n)-y(n) .
$$

$P(n)$ is the inverse correlation matrix at step $n$;

$$
P(n)=\Phi-1(n) .
$$

Here, our enhanced gain factor $k(n)$ is given as

$$
k(n)=\frac{\pi(n)}{m \times \lambda+u^{H}(n) \pi(n)},
$$

where $\pi(n)$ is denoted as

$$
\pi(n)=P(n-1) u(n)
$$

We initialize our algorithm by setting

Step (i) The weight vector, $\mathrm{w}(0)=0$

$$
\mathrm{P}(0)=\delta^{-1} \mathbf{I}
$$

Step (ii) And the value for $\delta$ depends on the SNR i.e,

$\delta=\left\{\begin{array}{l}\text { small positive constant for high } S N R \\ \text { large positive constant for low } S N R\end{array}\right.$.

Step (iii) The value of $\delta$ can be verified based on regularization grounds.

Step (iv) And for each instant of time, $n=1,2,3,, 4, \ldots$

Step (v) Compute:

$$
\begin{array}{r}
\pi(n)=P(n-1) u(n), \\
k(n)=\frac{\pi(n)}{m \times \lambda+u^{H}(n) \pi(n)} \quad \text { and } \\
P(n)=\frac{P(n-1)-k(n) * \pi(n)}{\lambda}
\end{array}
$$

The uniqueness of our improved algorithm only relates to improving the gain and directivity of the RLS algorithm as it enhances the design of the SA in a long range communication network. Therefore, we have enhanced the RLS algorithm by introducing a constant $m$ to the gain factor 
International Journal on AdHoc Networking Systems (IJANS) Vol. 8, No. 1, January 2018

$k(n)$ of the RLS algorithm. The result of this enhancement on the gain factor will influence the general performance of the weights at the output of the adaptive filter shown below.

The RLS algorithm is a deterministic algorithm in the sense that its performance index is the sum of weighted error squares for every given data. The weight vector is updated after every iteration.

The constant $m$ that has been introduced to the gain factor $k(n)$ will be given a value. We have conducted several tests in order to determine the best possible value of $m$ that can be introduced to $k(n)$ so that the weighted output of our RLS filter outperforms the weights of the other adaptive algorithms that have been implemented by smart antenna design.

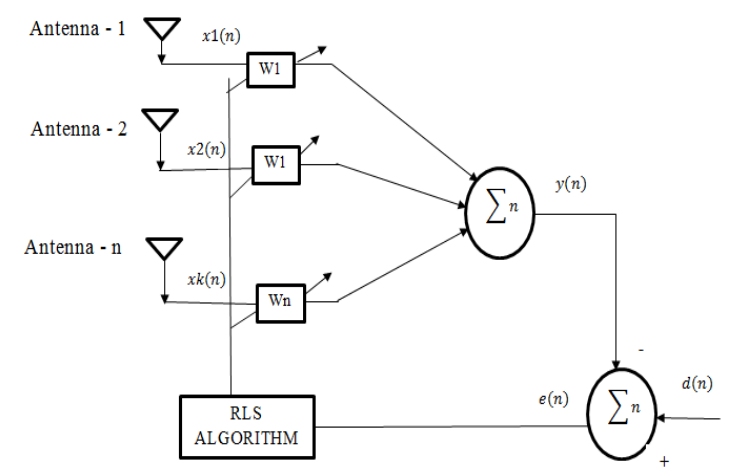

Figure 2: Computation of our RLS algorithm

\section{Performance Evaluation AND Results}

The performance of RLS based model for smart antenna design has been studied by means of MATLAB simulations. In these simulations, we have considered two cases taking into consideration the error curve and the system outputs of three adaptive algorithms namely: LMS, the conventional RLS and our enhanced RLS algorithm. The following parameters have been considered for simulation purposes:

TABLE I TYPE SIZE FOR PAPERS

\begin{tabular}{|c|l|c|l|}
\hline S/N & \multicolumn{1}{|c|}{ Initial Parameters } & Specification & \multicolumn{1}{c|}{ Reason } \\
\hline 1 & Number of antenna elements & 16 & $\begin{array}{l}\text { 16 elements are used so that our simulations will be } \\
\text { based on an antenna with optimum elements }\end{array}$ \\
\hline 2 & Number of Iterations & 1000 & $\begin{array}{l}\text { It is always advisable to conduct beamforming } \\
\text { simulations with more iteration. }\end{array}$ \\
\hline 3 & Forgetting factor & 0.99 & $\begin{array}{l}\text { A forgetting factor of 0.99 is a perfect value for the } \\
\text { RLS algorithm. }\end{array}$ \\
\hline 4 & Spacing between elements & 1 & Indicates the distance between each antenna element. \\
\hline 5. & Angle of transmitting antenna & $30^{\circ}$ & \\
\hline 6 & Angle of Interferer 1 & $-45^{\circ}$ & \\
\hline 7 & Angle of Interferer 2 & $45^{\circ}$ & \\
\hline
\end{tabular}

The results of the initial two simulations are discussed below. 
International Journal on AdHoc Networking Systems (IJANS) Vol. 8, No. 1, January 2018

Our aim is to see if the enhancment we have made on the gain factor $k(n)$ has an effect on the performance of the RLS algorithms. Firstly, we run simulations on the mean square errors (MSE). Based on the MSE curves in Fig. 3 ( $a, b$ and $c$ ), it can be observed that the error curve of the enhanced RLS algorithm dropped down to below $10^{-8}$ before 100 iterations. When compared to the error values of the conventional RLS algorithm, we see that the error value of the conventional RLS algorithm only drops down to $10^{-8}$ after 400 iterations. The LMS algorithm has a much higher error value (Fig. 3a) than both of the RLS algorithms. This result signifies lesser errors occurring in our enhanced RLS algorithm. Through these results, we can observe the superiority of the enhanced RLS algorithm over the LMS algorithm and the conventional RLS algorithm in terms of the mean square error.

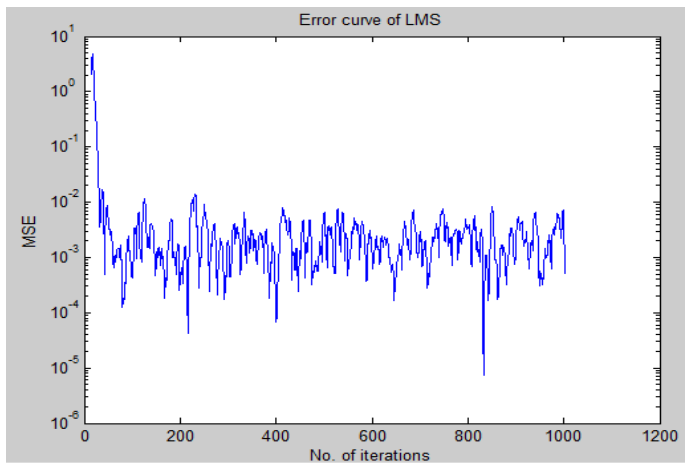

(a) LMS error

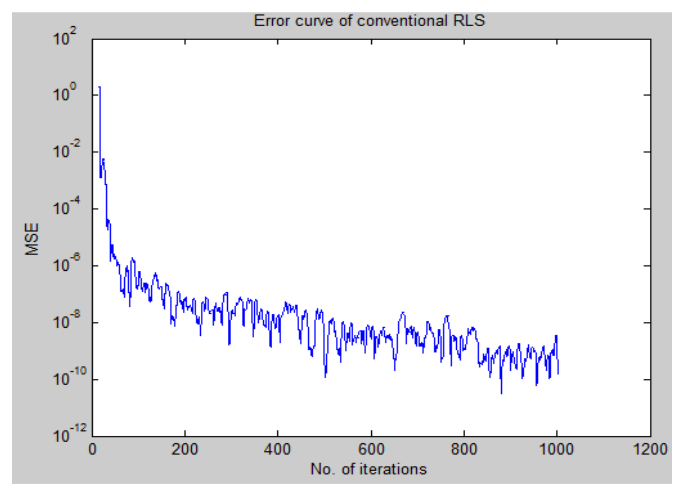

(b) Conventional RLS error

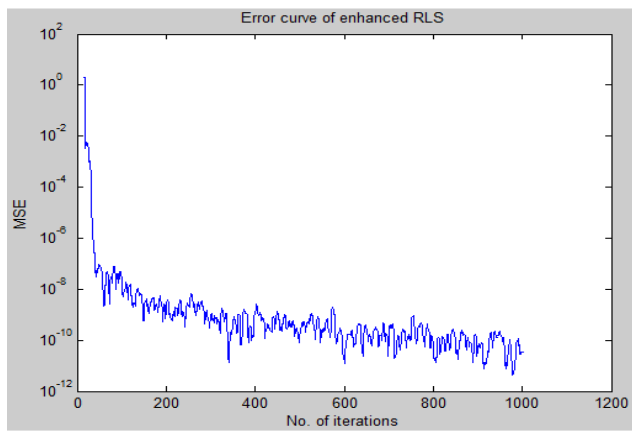

(c) Enhanced RLS error

Figure 3 : Error curve comparison 
International Journal on AdHoc Networking Systems (IJANS) Vol. 8, No. 1, January 2018

Secondly, our graphs in Fig. 4a, 4b and 4c respectively (below) show the system outputs of the LMS, conventional RLS and the enhanced RLS algorithms that are being compared. Based on these results, the following can be observed:

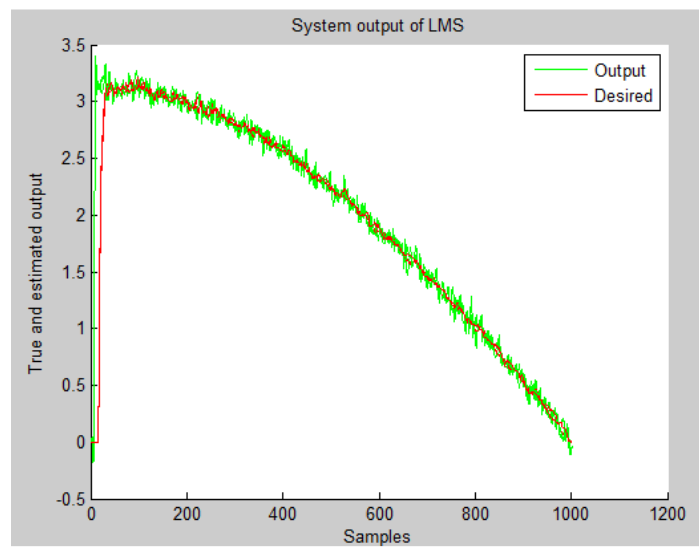

(a) LMS system output

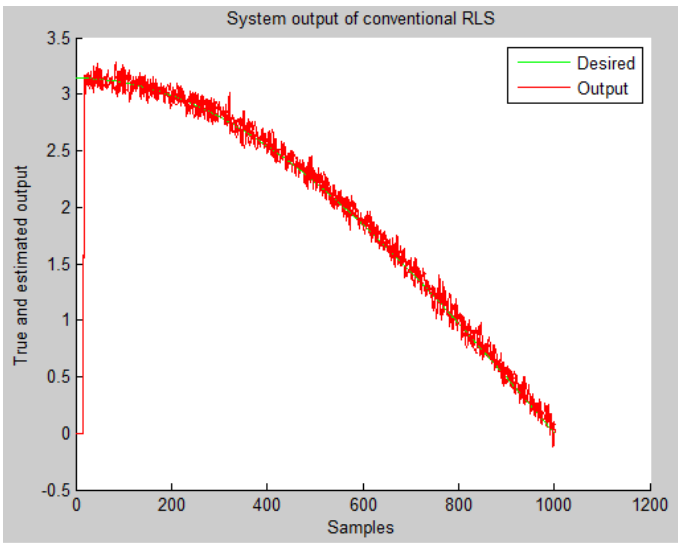

(b) Conventional RLS system output

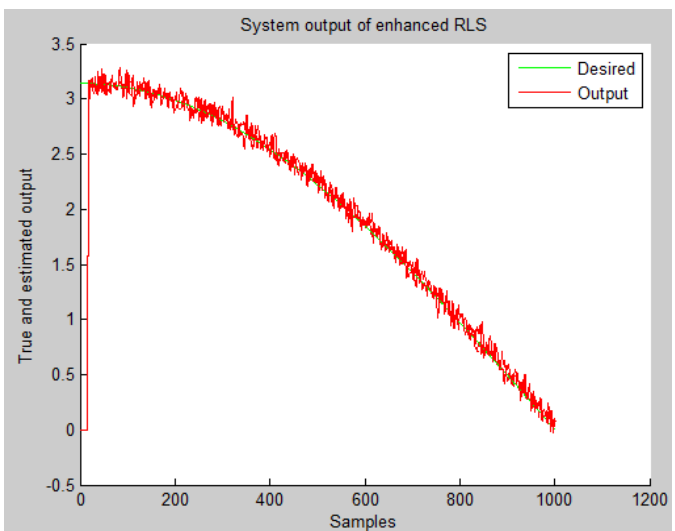

(c) Enhanced RLS system output

Figure 4 : Adaptive system output comparisons 
International Journal on AdHoc Networking Systems (IJANS) Vol. 8, No. 1, January 2018

The results of our simulations in Fig. 4 illustrate the outputs of the adaptive filters that we are testing namely: the LMS algorithm, the conventional RLS algorithm and our enhanced RLS algorithm. The results show that the enhanced RLS signals are less noisy compared to the other two algorithms. The output curve of our enhanced RLS algorithm is almost has the same curve as the desired output. This signifies a more filtered weights signal from our adaptive algorithm.

The last set of simulations takes into account what effects the number of antenna elements have on the shape of the beam. We conducted three simulations in Fig. 5 in order to see how the number of array elements $\mathrm{N}$ affects the directivity and narrowness of the resultant beam.

In Fig. 5a below, the resultant beam formed has some side lobes with connotes the presence of interfering signals. The beam is not narrow hence it is not so strong.
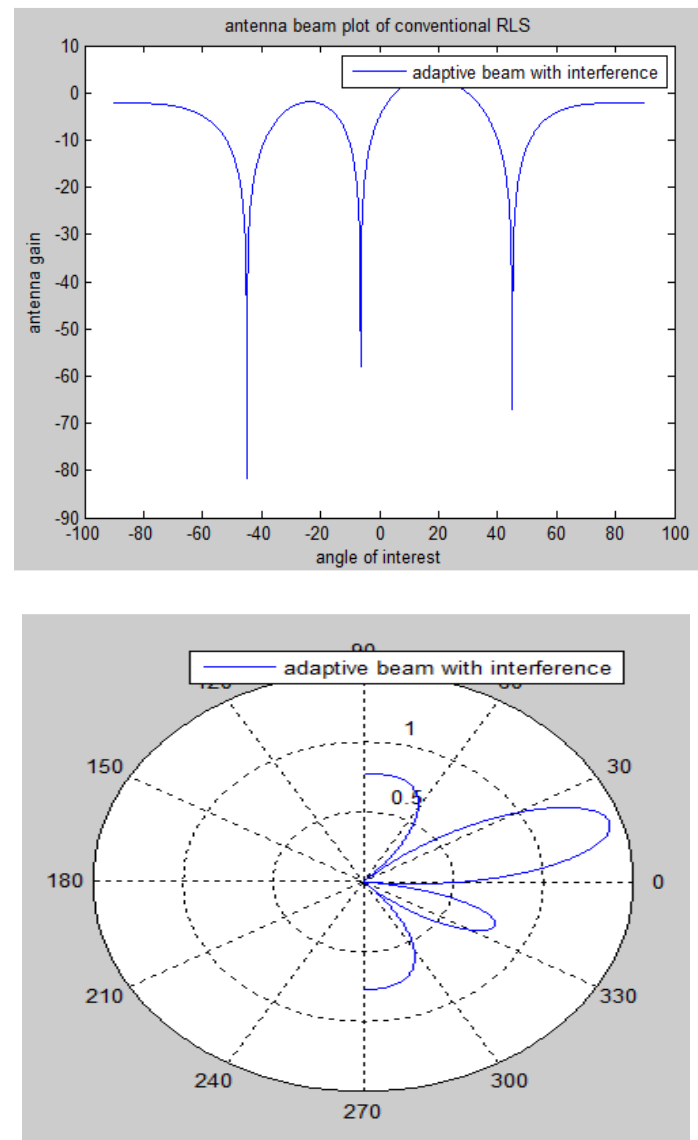

(a) Beam plots when $\mathrm{N}=4$ 
International Journal on AdHoc Networking Systems (IJANS) Vol. 8, No. 1, January 2018
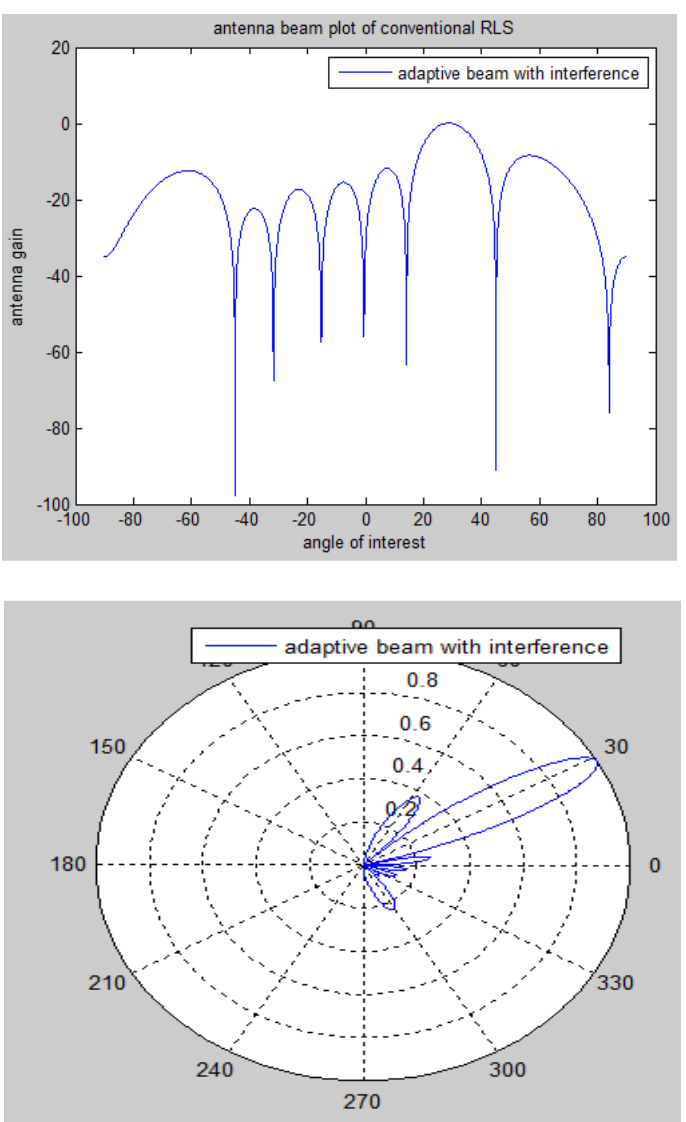

(b) Beam plots when $\mathrm{N}=8$

In Fig. $5 b$ above, the number of antenna elements $\mathrm{N}$ has been increased to 8 . We see that the resultant beam is more directed towards its user and the interfering signals have been reduced. Hence, the beam in fig. $5 \mathrm{~b}$ is stronger than that of Fig. $5 \mathrm{a}$.

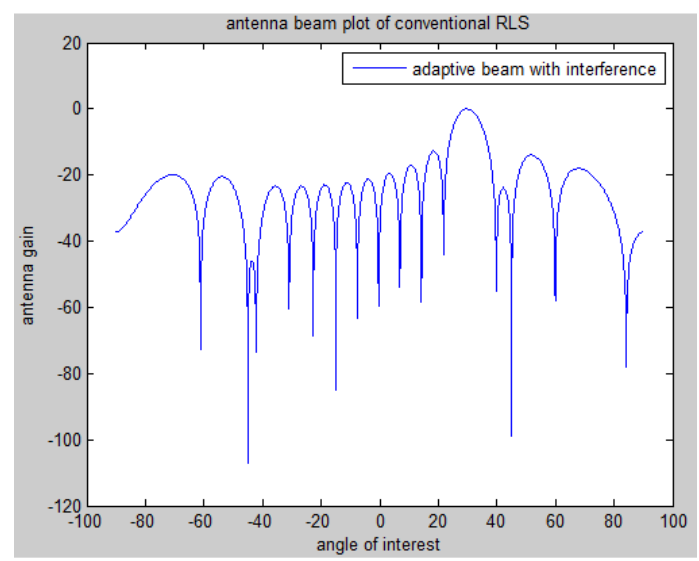


International Journal on AdHoc Networking Systems (IJANS) Vol. 8, No. 1, January 2018

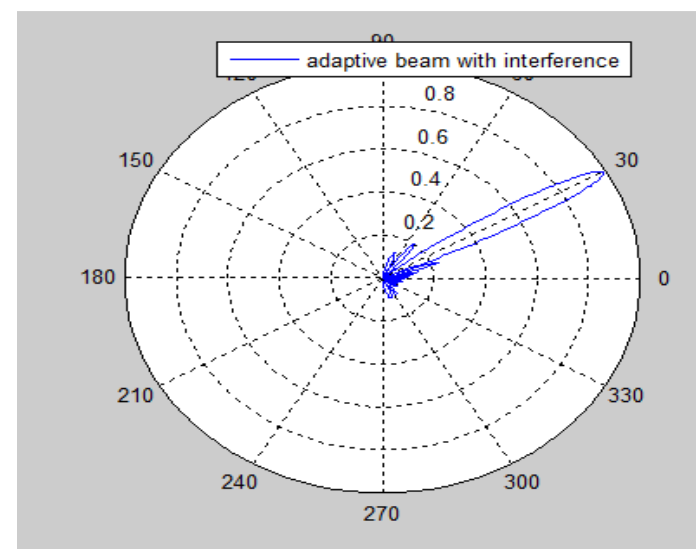

(c) Beam plots when $\mathrm{N}=16$

Figure 5 : Adaptive beams with interference

In fig. 5c, we increased the number of antenna elements to 16 . The results of our simulations show that the resultant beam is very narrow thus, indicating a very strong signal. It can also be seen that the interfering signals have been eliminated. Therefore, increasing the number of antenna elements has a positive impact in the strength and directivity of beamforming.

\section{CONCLUSION}

In this paper, the performance evaluation for beamforming over long range communication channel is discussed. We have enhanced the gain factor $k(n)$ of the RLS algorithm in order to improve its performance which results to an increase in the gain and the directivity of the smart antenna. A constant $m$ has been introduced to the gain factor $k(n)$ of the RLS algorithm with an expectation of enhancing the performance of the output of the adaptive antenna. Results of the simulations carried out in figures 3 and 4 show the following: the comparison of MSE and the comparison of the system outputs of the different adaptive algorithms. These simulations show that the enhanced RLS algorithm possesses better performance in terms of lower MSE (Fig. 3c) which connotes a quicker rate of convergence of the adaptive algorithm. Also, the output of the enhanced RLS algorithm shows a smoother waveform thus indicating that it is the best adaptive filter; thus eliminating unwanted noise from surrounding communication networks.

We further conducted some simulations to illustrate the effects of the number of antenna elements $\mathrm{N}$. When the number of antenna element is increased, the resultant beam possesses a stronger directivity and is less susceptible to interfering signals.

\section{ACKNOWLEDGMENT}

The authors would like to acknowledge Tshwane University of Technology for providing research facilities and equipment for this study. 
International Journal on AdHoc Networking Systems (IJANS) Vol. 8, No. 1, January 2018

\section{REFERENCES}

[1] Balanis, Antenna Theory: Analysis and Design, (New Jersey). John Wiley and sons, C.A. 2005.

[2] S. Verma and A. Pathak: "Digital Beamforming Using RLS-QRD Algorithm". International Journal of Engineering Research and Technology (IJERT), ISSN: 2278-0181, Vol. 1, Issue 2, July 2012.

[3] N.A. Mohamed and J. G. Dunham: "Adaptive Beamforming for DS-CDMA using Conjugate Gradient Algorithm in a Multipath Fading Channel”. Emerging Technologies Symposium on Wireless Communications and Systems, pp. 1.1-1.5, Richardson, TXUSA. April, 1999.

[4] J. A. Stine: "Exploiting Smart Antennas in Wireless Mesh Networks Using Contention Access". IEEE Trans. on Wireless Communications, vol. 13, pp. 38-49, 2006.

[5] B. S. Reddy, A. S. Bhalchandra and V. R. Ratnaparkhe: "Adaptive Digital Beamforming Using LMS Algorithm”. IOSR Journal of Electronics and Communication Engineering. e-ISSN: 2278-2834, pISSN: 2278-8735. Volume 9, issue 2, ver. IV (March - April 2014), pp 63-68.

[6] S. Das: "Smart Antenna Design for Wireless Communication Using Adaptive Beamforming Approach".

[7] R. Joshi and A. Dhande: “Adaptive Beamforming Using LMS Algorithm”. International Journal of Research in Engineering and Technology; vol. 3, Issue 05. May 2014.

[8] L. C. Godara: “Application of Antenna Arrays to Mobile Communications, Part II: Beamforming and Direction-of-Arrival Considerations”. Proceedings of IEEE, vol. 85, no. 8, pp. 1195-1245. 1997

[9] Simon Haykin; "Signal processing: Where Physics and Mathematics meet". IEEE Signal Processing Magazine, 18(4): 6-7; 2001.

[10] Laboratory Technique Spatial Filtering: http://cem01.ucsd.edu/ vitaliy/courses/ece182/18206files/SpatialFiltering.pdf

[11] A. Khan: "Beamforming using Adaptive Algorithms". BS Telecommunications; Session 2010-2014.

[12] I. Lie and M.E Tanase: "A Compact FPGA Beamformer Architecture”. 2005 WSEAS International conference on Dynamical sytems and control. Pp 463- 466; Venice, Italy. November 2-4, 2005.

[13] Laboratory Technique Spatial Filtering: http://cem01.ucsd.edu/ vitaliy/courses/ece182/18206files/SpatialFiltering.pdf.

[14] Antenna Arrays and Beamforming http://scholar.lib.vt.edu/theses/available/etd-0426200015330030/unrestricted/ch3.pdf

[15] xhttp://en.wikipedia.org/wiki/Adaptive

[16] P. M. Mainkar, G. N. Mulay and S. C. Upadhyay: "Performance Evaluation of the RLS Adaptive Beamforming Algorithm”. ISSN 2319-9229, Vol. 1, issue 6, April 2013.

[17] G. V. Tsoulos; Smart antennas for mobile communication systems: benefits and challenges. Electronics \& Communication Engineering Journal, April 1999. 
International Journal on AdHoc Networking Systems (IJANS) Vol. 8, No. 1, January 2018

[18] J. A. Srar and K. S. Chung: "Adaptive Array Beamforming Using a Combined RLS-LMS Algorithm". Proceedings of APCC 2008.

[19] L. C. Godara: "Application of Antenna Arrays to Mobile Communications, Part II: Beamforming and Direction of Arrival Considerations". Proceedings of IEEE, vol. 85, no. 8, pp. 1195-1245.

[20] C. A. Balanis: “Antenna Theory: Analysis and Design”. New Jersey: John Wiley and Sons, 2005.

[21] B. Farhang-Boroujeny: "Adaptive Filters Theory and Applications". John Wiley and Sons, New York, 1998. 